\title{
Communicating with Persons Who Express Spiritual Struggle at the End of Life
}

\author{
Elizabeth Johnston Taylor, R.N., Ph.D., FAAN \\ School of Nursing, Loma Linda University, Loma Linda, CA, USA
}

This paper provides practical suggestions for how palliative care clinicians can address the expressions of spiritual struggle voiced by patients and their loved ones. In addition to practical tips for listening and responding, ethical guidance and opportunities for self-reflection related to spiritual care are briefly discussed. Principles to guide practice when the clinician is listening and responding to a patient expressing spiritual struggle include being nondirective, honoring (vs. judging) the patient's spiritual or religious experience, keeping the conversation patient-centered, focusing on the core theme of what the patient is expressing presently, using the patient's terminology and framing, and responding "heart to heart" or "head to head" to align with the patient. Ultimately, the goal of a healing response from a spiritual care generalist is to allow the patient to "hear" or "see" themselves, to gain selfawareness. To converse with patients about spirituality in an ethical manner, the clinician must first assess the patient's spiritual needs and preferences and then honor these.

Correspondence to

Elizabeth Johnston Taylor ORCID:

https://orcid.org/0000-0002-6790-8801 E-mail: ejtaylor@llu.edu

\section{COMMUNICATING WITH PERSONS WHO EXPRESS SPIRITUAL STRUGGLE AT THE END OF LIFE}

Spiritual struggle among persons facing the end of their life is manifest in many ways. Often the struggle is expressed with words. For example:

- Why does this have to happen to me now?

- I feel like such a burden to my family.

- I used to think there was a God who was loving and there for me. Now I don't know what to believe.

- Things don't always work out for the best. That angers me!

Although a life-threatening disease can prompt spiritual growth, renewed commitments to religious practices, and questing to make life and relationships more meaningful, it often also produces spiritual struggle, pain, or distress. Indeed, spiritual growth and struggle are often concurrent. The varied facets of spiritual responses to a life-threatening disease among Koreans parallel those experienced by others [1]. Furthermore, evidence indicates that the spiritual needs and struggles of terminally ill patients are mirrored in the experiences of their primary family carers [2].

Thus, the purpose of this paper is to provide practical suggestions for how palliative care clinicians can address the expressions of spiritual struggle voiced by patients and their loved ones. In particular, the focus will be on how clinicians can converse (i.e., deeply listen and verbally respond) to patients and family members in this context. In addition to practical tips for listening and responding, ethical guidance and opportunities for self-reflection related to spiritual care will be briefly discussed. 


\section{COMMUNICATION SKILLS FOR SPIRITUAL CARE}

Healthcare professionals learn basic therapeutic communication skills in their pre-licensure training; however, these skills are often inadequately mastered and difficult to apply when a patient expresses spiritual struggle and consequent difficult emotions. It is as though hearing such suffering ties the tongue in a knot! Actually, it creates fear within the clinician (e.g., "maybe this will happen to me" or "maybe this will overwhelm me") [3].

When clinicians hear a patient's spiritual discomfort that causes their own inner discomfort, they typically avoid it. There are several ways a clinician can avoid remaining present to spiritual pain [3]. They may:

- Change the topic (e.g., ask about the family's perspective instead of the patient's, ask about technicalities or irrelevant facts, or move the conversation to a completely different topic)

- Minimize the patient's suffering (e.g., "It couldn't really be that bad" or interject humor)

- Impose a positive perspective (e.g., "At least something good has happened as a result")

- Give a superficial answer or "quick fix" (e.g., "There's a reason for it" or "Just trust it will all work out").

When the clinician consistently avoids the patient's spiritual pain, the patient learns it is not safe to discuss this very sensitive topic with this professional. The patient will be silenced; an opportunity for healing will be denied. Why be vulnerable if only to be rebuffed or disregarded? Patients, however, often give clinicians some grace; they may broach the topic a few times before giving up on a clinician [3].

Assuming the clinician is willing to remain present to a patient who is expressing spiritual pain, there are several principles to guide a therapeutic verbal response $[3,4]$. These are as follows:

- Be non-directive. Avoid controlling the conversation. The goal is not to direct the patient to the spiritual/religious (S/ R) beliefs that you think they need to adopt. Rather, the goal is to allow the patient to "hear" or "see" themselves, to gain self-awareness. Being able to vulnerably give expression to the deepest self and having a loving other respond in ways that allow one to hear and see themselves more accurately allows one to explore inwardly and fosters self-awareness. If the exploration leads to insight, then it may also lead to decisions or actions that align. Basic communication skills of offering restatements, paraphrasing, and summarizing allow a clinician to be nondirective. Open questions that are not leading will also be helpful if not overused or framed in an accusatory manner.

- Honor. Respect (vs. judge) the patient's S/R experience. After all, every person has a unique experience that contributes to a beautiful human kaleidoscope. Also, even "negative" S/R experiences are graces that can generate inner maturation. Practically, this means that clinicians need to maintain neutrality in their responses. Even responding with affirmations that show approval of $\mathrm{pa}^{-}$ tients' S/R experiences may convey to the patient that they need to say the right things to keep receiving approval. For example, "It is so good that you pray/meditate every day; I' $\mathrm{m}$ sure that helps you" makes it difficult for the patient to then discuss the spiritual struggle that ensues from feeling like God is absent during the prayers or that they feel like they are bad at meditating. Notice that a self-disclosure delivered for the purpose of building rapport or communicating compassion has a different quality. For example, "I'm praying for you" is typically well-received in American culture. It communicates care rather than judgment.

- Keep conversation patient-centered, or in patient's "court." This metaphor refers to the game of tennis, where the objective is to volley the ball back to the other person. So it is with a therapeutic conversation, given the focus is not on the clinician but rather on the patient. Thus, keep your responses short. Refrain from telling personal stories unless it is for therapeutic reasons (e.g., to illustrate, to decrease the patient's anxiety, to build rapport). If selfdisclosing, ask yourself, "For who am I self-disclosing?" If it is to self-comfort, to impose what you believe is truth, to brag, or for some other personal need, then refrain from the self-disclosure. After self-disclosure, return the conversation to the patient's court (e.g., "That's my story; I'm wondering what thoughts or feelings it generates inside you?”). 
- Focus on core theme of what the patient is expressing now. Whether the patient is communicating verbally or non-verbally, consider what is the "cutting edge" or most vital element of what they are saying. Often the core theme is that which is talked about with the most emotional or physical energy (e.g., eyes tearing or beaming, voice slowing or quickening). The core theme is also often described with a metaphor (e.g., "I feel like a ship without a sail" or "I wish they'd pull the plug on me"). Since it is so challenging to remain present to $S / R$ pain, it is easy to avoid it with responses that focus on a tangent (e.g., "How is this affecting your family?" [when it is a personal struggle that is causing the patient anguish] or "What kind of cancer did the doctor say you have?").

- Use patient's S/R language. Especially when discussing S/ $\mathrm{R}$ issues, it is important to show respect for the patient's worldview as reflected in their language. A non-religious patient may talk about their desire to develop inner harmony or to be at peace at the end of life. Or they may describe how beauty and love give life meaning. Religious persons with some type of theistic belief could have any number of names for the divine (e.g., Sacred Source, Higher Power, God, Buddha). Note the patient's use of terms and $\mathrm{S} / \mathrm{R}$ framings and avoid imposing your own into conversations.

- Talk "heart to heart," or "head to head." When listening to others holistically, the clinician will listen to not just the facts, stories, and ideas (i.e., the intellectual content), but also to emotional and physical elements. For example, consider what feelings are crying out in the para-language and what messages the body language gives. If the patient is communicating in a way that expresses considerable emotion (e.g., tears, vocal change, feelings verbally expressed), the therapeutic clinician will respond in a way that reflects back these feelings. Such responses use empathy and very tentatively name the type of feeling and its intensity (e.g., "I'm guessing you're feeling like a burden and sorrowful because you can no longer walk by yourself.") As this example illustrates, empathic responses not only tentatively name the feeling, but also often link it to the situation causing it. When a patient is talking from the "head," a therapeutic response that is intellectual is best (e.g., "What thoughts do you have about that?"). Thus, helpful head-oriented responses include restatements (and similar techniques) and open questions that are not about feelings, whereas helpful heart-oriented responses include empathic reflections of feelings and open questions.

Clinicians providing healthcare are unable to provide any in-depth psychospiritual therapy for patients with spiritual pain. Not only do they not have adequate training (i.e., they are spiritual care generalists, not specialists), but they typically do not have much time. They may have only a few minutes, at best, to listen to and respond to spiritual pain. Thus, it is important to remember that the goal for these conversations is to allow the process of inner exploration to begin. Essentially, a clinician should apply the principles presented to create a therapeutic milieu whereby patients can hear and see themselves.

\section{ETHICAL GUIDANCE}

Given the elemental nature of S/R beliefs to personhood, the exquisite sensitivity required for therapeutically addressing another's spiritual struggle, and the social taboos that often accompany discussions of $S / R$ phenomena in most cultures, ethical principles ought to guide such spiritual care. The concern is that the clinician will approach such conversations with inappropriate motives or inadequate skill. The clinician could overtly proselytize or unwittingly impose personal beliefs and practices; such unethical clinician behavior would be an abuse of the power differential within the clinician-client relationship [5].

Thus, the ethicist Winslow and nurse scholar Wehtje-Winslow offered the following guidelines for ensuring that spiritual care is ethical [6]. These simply stated considerations are easily implemented by any clinician. The ethical guidelines include:

a) Screen for and/or assess the patient's (or family carer's) spiritual needs, resources, and preferences (e.g., What S/ $\mathrm{R}$ practices would they like to have supported or respected while hospitalized? In what ways would they like the staff to honor their $\mathrm{S} / \mathrm{R}$ beliefs or practices?)

b) Honor the patient/family's wishes about receiving spiritual care.

c) Never impose your S/R beliefs or practices on the patient/ 
family; likewise, never pressure them to relinquish theirs [6]. If the clinician lacks an awareness of personal S/R beliefs and practices and how it influences the spiritual care they provide, however, then these three steps are difficult. Thus, the Winslows advocated that clinicians develop spiritual self-awareness. They also posited that ethical S/R care means caring in ways that honor the clinician's S/R beliefs and practices.

An additional point to consider is that patients often do not expect or want their healthcare providers to address spiritual or religious needs. Religious persons and those who perceive their illness as very serious (e.g., terminal), however, are more welcoming of clinicians' inquiry and attention to spiritual concerns [3]. The findings in this regard, however, are sparse and varied. For example, in an American sample of outpatients with heart failure, 54\% of 111 responded not at all to an item asking whether they wanted their "doctor or other healthcare providers to attend to your spiritual needs" [7]. In contrast, $63 \%$ of 94 terminally ill patients receiving care at a Korean palliative care center reported that they believed it was important for the healthcare team members to address $S / R$ needs and 50.5\% reported that their S/R needs had been met [8].

Since not all patients will expect or want to discuss their spiritual struggles with a healthcare provider, it is important to facilitate care from a healthcare chaplain or communitybased clergy or spiritual healer of the patient's choosing. The clinician might assess this need with a question like, "Would a spiritual care expert be helpful to you as you think about these difficult spiritual questions?" and "Is there someone you already know who you can trust would be helpful?" It is my observation from personal experience and from interviews with nurse experts in spiritual care that if rapport is established and the clinician indicates willingness and safety, patients will typically pour their "souls" out [9]. The issue is usually not getting a patient to disclose their spiritual struggles, but having clinicians respond therapeutically.

\section{SELF-REFLECTION}

As Winslow and Wehtje-Winslow noted [6], clinicians' S/ $\mathrm{R}$ self-awareness is paramount for ethical spiritual care. To be able to hear a patient's spiritual struggle requires that a clinician have some awareness of how they, too, have struggled spiritually [3]. If we avoid our own S/R questions, doubts, and dilemmas, we will avoid those expressed by patients. Whereas we do not need to have had the same experiences, we can identify with the same emotions and let those inform our therapeutic responses. For example, a clinician may not have grieved the loss of a child, but will have grieved for someone or something (e.g., a pet, relationship, role, youth, status). Remembering this grief will allow the clinician to remain present and sensitive to another's grief.

Self-reflection can become part of regularly scheduled selfcare. Depending on one's personality, the clinician can studiously ponder and keep a diary about questions such as those that follow, use finger painting (or other expressive art form), or engage in another method to explore their inner life. The following questions may be helpful prompts:

- What S/R questions do I hear from patients that really make me avoid them? What $S / R$ struggles would I have if I were experiencing chronic, debilitating, or terminal illness? What S/R doubts would I have if I were facing the end of my life?

- Is there one truth only to answer S/R questions and struggles? Or can a myriad of $\mathrm{S} / \mathrm{R}$ responses be a manifestation of divine creativity? How might this epistemological belief impact the way I respond to patients?

- What are my worst fears and anxieties? What most angers me? What do I do with "negative" feelings? What do they indicate about my thoughts? How can I accept them as gifts that teach? What do they remind me about in my upbringing or life experiences that I am still integrating?

- When I reflect about a patient encounter that I "ran away" from, what did I most deeply fear? Why? What would I do differently now?

Regardless of what prompts or methods bring fruitful selfawareness, it is not only the clinician who will benefit. Patients will also be recipients, because this self-reflection will allow the clinician to more effectively be a healing presence to $\mathrm{pa}^{-}$ tients.

\section{CONFLICT OF INTEREST}

The author acknowledges that royalties are received for a publication cited multiple times within this manuscript. 


\section{REFERENCES}

1. Mamier I, Kim SM, Petersen D, Bae HJ, Taylor EJ, Kang KA. Spiritual needs among Koreans and Americans with advanced chronic illnesses: a cultural comparison. J Clin Nurs 2021;30:3517-27.

2. Kim SS, Hayward RD, Kang Y. Psychological, physical, social, and spiritual well-being similarities between Korean older adults and family caregivers. Geriatr Nurs 2013;34:35-40.

3. Taylor EJ. What do I say? : talking with patients about spirituality. West Conshohocken, PA:Templeton Foundation;2007.

4. Hill CE. Helping skills: facilitating exploration, insight, and action. 5th ed. Washington, DC: American Psychological Association;2020.

5. Taylor EJ. Religion: a clinical guide for nurses. New York:Springer;2012.

6. Winslow GR, Wehtje-Winslow BJ. Ethical boundaries of spiritual care. Med J Aust 2007;186(S10):S63-6.

7. Park CL, Sacco SJ. Heart failure patients' desires for spiritual care, perceived constraints, and unmet spiritual needs: relations with wellbeing and health-related quality of life. Psychol Health Med 2017;22:1011-20.

8. Kang J, Shin DW, Choi JY, Park CH, Baek YJ, Mo HN, et al. Addressing the religious and spiritual needs of dying patients by healthcare staff in Korea: patient perspectives in a multi-religious Asian country. Psychooncology 2012;21:374-81.

9. Pfeiffer JB, Gober C, Taylor EJ. How Christian nurses converse with patients about spirituality. J Clin Nurs 2014;23:2886-95. 